ARTICLE

\title{
Cobalt-centred boron molecular drums with the highest coordination number in the $\mathrm{CoB}_{16} \bar{c}$ cluster
}

Ivan A. Popov ${ }^{1, \star}$, Tian Jian ${ }^{2, \star}$, Gary V. Lopez ${ }^{2}$, Alexander I. Boldyrev ${ }^{1}$ \& Lai-Sheng Wang ${ }^{2}$

The electron deficiency and strong bonding capacity of boron have led to a vast variety of molecular structures in chemistry and materials science. Here we report the observation of highly symmetric cobalt-centered boron drum-like structures of $\mathrm{CoB}_{1 \overline{6}}$, characterized by photoelectron spectroscopy and $a b$ initio calculations. The photoelectron spectra display a relatively simple spectral pattern, suggesting a high symmetry structure. Two nearly degenerate isomers with $D_{8 d}$ (I) and $C_{4 v}$ (II) symmetries are found computationally to compete for the global minimum. These drum-like structures consist of two $B_{8}$ rings sandwiching a cobalt atom, which has the highest coordination number known heretofore in chemistry. We show that doping of boron clusters with a transition metal atom induces an earlier two-dimensional to three-dimensional structural transition. The $\mathrm{CoB}_{16}$ cluster is tested as a building block in a triple-decker sandwich, suggesting a promising route for its realization in the solid state.

\footnotetext{
${ }^{1}$ Department of Chemistry and Biochemistry, Utah State University, Logan, Utah 84322, USA. ${ }^{2}$ Department of Chemistry, Brown University, Providence, Rhode Island 02912, USA. * These authors contributed equally to this work. Correspondence and requests for materials should be addressed to A.I.B. (email: a.i.boldyrev@usu.edu) or to L.-S.W. (email: lai-sheng_wang@brown.edu).
} 
B oron, the fifth element in the periodic table, possesses such diverse chemical structures and bonding that are second only to carbon. Bulk boron consists of connected three-dimensional (3D) cages in many of its allotropes ${ }^{1,2}$ and boron-rich borides $^{3,4}$. However, for isolated clusters it was computationally shown $n^{5,6}$ that icosahedral cage structures of $B_{12}$ and $B_{13}$ were unstable, even though they were initially proposed as possible candidates for these two clusters ${ }^{7}$. Over the past decade, small anionic boron clusters have been systematically characterized both experimentally and theoretically to exhibit planar or quasi-planar structures in their ground states up to $\mathrm{B}_{27}^{-}$(refs $8-10$ ). Recent works show that anionic boron clusters continue to be two-dimensional (2D) at $\mathrm{B}_{30}^{-}$(ref. 11), $\mathrm{B}_{35}^{-}$(ref. 12) and $\mathrm{B}_{36}^{-}$(ref. 13). The 2D-to-3D transition was suggested to occur at $\mathrm{B}_{20}$ for neutral ${ }^{14}$, and at $\mathrm{B}_{16}^{+}$for cationic clusters ${ }^{15}$. Very recently it is shown that the transition from $2 \mathrm{D}$ to fullerene-like $3 \mathrm{D}$ structures occurs in negatively charged boron clusters at about 40 boron atoms in $\mathrm{B}_{39}^{-}$(ref. 16) and $\mathrm{B}_{40}^{-}$(ref. 17). Due to the nearly spherical shapes of these clusters, they have been named borospherenes. Doping boron clusters with a single metal atom opens a new avenue to create clusters with novel structures and chemical bonding. It has been experimentally observed that various transition metal atoms can be placed inside of monocyclic boron rings to form beautiful molecular wheel-type structures $\left(\mathrm{M} C \mathrm{~B}_{n}^{-}\right)^{18}$, following an electronic design principle inspired by the doubly $\sigma$ and $\pi$ aromatic $\mathrm{B}_{9}^{-}$cluster $^{19}$. It was shown that the $\mathrm{Nb} C \mathrm{~B}_{10}^{-}$and $\mathrm{Ta} C \mathrm{~B}_{10}^{-}$clusters possess the record coordination number of 10 in the planar environment for the central metal atom ${ }^{20}$. These clusters have pushed the limits of structural chemistry.

Here we report the observation of a large metal-doped boron cluster of $\mathrm{CoB}_{16}^{-}$, which is produced using a laser vaporization cluster source and characterized by photoelectron spectroscopy (PES). Extensive computational searches reveal that there are two nearly degenerate structures for $\mathrm{CoB}_{16}^{-}$, which are indistinguishable at the highest level of theory employed. They both possess tubular double-ring framework and give similar photoelectron spectral patterns. The structures can be viewed as two $\mathrm{B}_{8}$ rings sandwiching a Co atom, reminiscent of a drum and giving rise to the highest coordination number known in chemistry thus far.

\section{Results}

Experimental results. The photoelectron spectra of $\mathrm{CoB}_{16}^{-}$at two photon energies are displayed in Fig. 1. The lowest binding energy band $(\mathrm{X})$ represents the electron detachment transition from the anionic ground state to that of neutral $\mathrm{CoB}_{16}$. The higher binding energy bands, $A, B, \ldots$, denote detachment transitions to the excited states of neutral $\mathrm{CoB}_{16}$. The vertical detachment energies (VDEs) for all observed bands are given in Table 1, where they are compared with the calculated VDEs.

The $266 \mathrm{~nm}$ spectrum (Fig. 1a) reveals three well-resolved PES bands for $\mathrm{CoB}_{16}^{-}$. The band $\mathrm{X}$ gives rise to a VDE of $2.71 \mathrm{eV}$. The adiabatic detachment energy (ADE) for band $\mathrm{X}$ was evaluated from its onset to be $2.48 \mathrm{eV}$, which also represents the electron affinity of neutral $\mathrm{CoB}_{16}$. The width of band $\mathrm{X}$ suggests an appreciable geometry change between the ground electronic state of $\mathrm{CoB}_{16}^{-}$and the ground electronic state of $\mathrm{CoB}_{16}$. Following a relatively large energy gap, an intense and broad band $\mathrm{A}$ is observed at a VDE of $3.45 \mathrm{eV}$ and a close-lying band B at a VDE of $3.78 \mathrm{eV}$. The $193 \mathrm{~nm}$ spectrum (Fig. 1b) shows nearly continuous signals beyond $4 \mathrm{eV}$. The sharp spikes above $5 \mathrm{eV}$ in the high binding energy side of the $193 \mathrm{~nm}$ spectrum are due to statistical noises because of low electron counts. An intense and broad band $\mathrm{C}$ is clearly observed at a VDE of $4.86 \mathrm{eV}$. Two more bands can be tentatively identified at higher binding energies, D (VDE: $\sim 5.3 \mathrm{eV}$ ) and E (VDE: $\sim 5.6 \mathrm{eV}$ ). Overall, the PES spectral

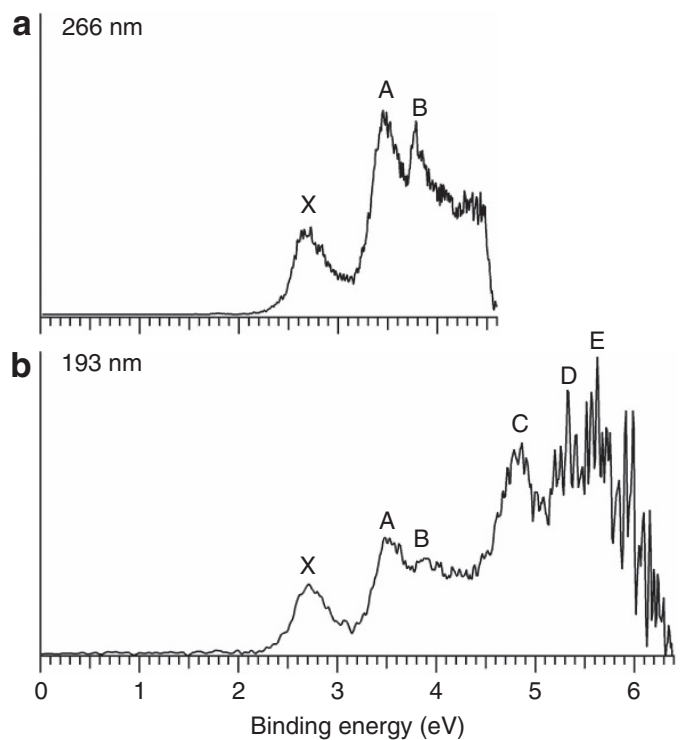

Figure 1 | Photoelectron spectra. Photoelectron spectra (a) at $266 \mathrm{~nm}$ $(4.661 \mathrm{eV})$ and $(\mathbf{b})$ at $193 \mathrm{~nm}(6.424 \mathrm{eV})$ of $\mathrm{CoB}_{16}$.

pattern is relatively simple, suggesting that the framework of the

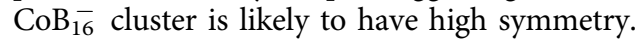

Theoretical results and comparison with experiment. Extensive structural searches were initially done at the PBE0/3-21G level of theory with the follow-up calculations $\left(\Delta=25 \mathrm{kcal} \mathrm{mol}^{-1}\right)$ at the PBE0/Def2-TZVP level of theory, which led to two similar drumlike structures: isomer I $\left(\mathrm{D}_{8 \mathrm{~d}},{ }^{3} \mathrm{~A}_{2}\right)$ and isomer II $\left(\mathrm{C}_{4 \mathrm{v}},{ }^{1} \mathrm{~A}_{1}\right)$ identified as the global minima for $\mathrm{CoB}_{16}^{-}$(Fig. 2). These two highly symmetric structures, consisting of a central Co atom sandwiched by two $\mathrm{B}_{8}$ monocyclic rings, are found to be almost degenerate at various levels of theory (Supplementary Fig. 1 and Supplementary Table 1). Clearly, the method dependency of predicting relative energies of the low-lying structures for $\mathrm{CoB}_{16}^{-}$ suggests the importance of comparison with experiment in determining the global minimum. We previously studied how optimized geometries of small boron clusters differed at density functional theory (DFT) and $\operatorname{CCSD}(\mathrm{T})$ levels of theory ${ }^{21,22}$. We found that B3LYP/6-311 $+\mathrm{G}^{*}$ geometries are quite close (within $0.03 \AA$ between nearest boron atoms) to those at the $\operatorname{CCSD}(\mathrm{T}) /$ $6-311+\mathrm{G}^{*}$ level of theory. We also compared the geometries of boron clusters at PBE0/6-311 $+\mathrm{G}^{*}$ and B3LYP/6-311 $+\mathrm{G}^{*}$, and found that they are also very close ${ }^{23}$. Therefore, PBE0/3-21G level of theory was used for the preliminary search and PBE0/Def2TZVP for the final optimized geometries of $\mathrm{CoB}_{16}^{-}$. The highest level of theory employed (ROCCSD(T)/6-311+ G(2df)//PBE0/ Def2-TZVP (this abbreviation means that single-point energy calculations were performed at $\operatorname{ROCCSD}(\mathrm{T}) / 6-311+\mathrm{G}(2 \mathrm{df})$ using optimized UPBE0/Def2-TZVP geometries here and elsewhere) indicates $1.4 \mathrm{kcal} \mathrm{mol}^{-1}$ energy difference including zero-point energy corrections (Supplementary Fig. 1). This small value is in the range of the theoretical errors for such a complex transition-metal-doped boron cluster. Therefore, isomers I and II should be considered to be degenerate based on our calculations. Figure 2 shows the small differences in bond distances between isomers I and II; the latter is not significantly distorted from the $\mathrm{D}_{8 \mathrm{~d}}$ symmetry. The $\mathrm{B}-\mathrm{B}$ bond lengths of the $\mathrm{B}_{8}$ rings for both isomers are in the range of 1.55-1.63 $\AA$, similar to the corresponding values $(1.56 \AA)$ in the $\mathrm{Co} C \mathrm{~B}_{8}^{-}$molecular wheel ${ }^{18}$. The nearest isomer III $\left(\mathrm{C}_{2},{ }^{1} \mathrm{~A}\right)$ is $8.7 \mathrm{kcal} \mathrm{mol}^{-1}$ higher in energy at the $\operatorname{ROCCSD}(\mathrm{T})$ method and represents a 
Table 1 | Experimental and theoretical vertical electron detachment energies (VDEs) in $\mathrm{eV}$ of $\mathrm{CoB}_{\mathbf{1 6}}$.

\begin{tabular}{|c|c|c|c|c|c|c|c|c|c|c|c|c|c|}
\hline & \multirow[t]{3}{*}{$\begin{array}{c}\text { VDE } \\
\left(\text { exp.) }{ }^{\star}\right.\end{array}$} & \multicolumn{6}{|c|}{$\begin{array}{c}\text { Isomer I } \\
\begin{array}{c}\left(1 a_{1}{ }^{2} 1 e_{1}^{4} 1 e_{2}{ }^{4} 1 b_{2}{ }^{2} 1 e_{3}^{4} 2 e_{1}^{4} 2 a_{1}{ }^{2} 1 e_{4}^{4} 2 e_{2}{ }^{4}\right. \\
\left.3 a_{1}{ }^{2} 3 e_{2}{ }^{4} 2 e_{3}{ }^{4} 3 e_{1}^{4} 4 e_{1}^{4} 5 e_{1}^{4} 4 a_{1}{ }^{2} 2 b_{2}{ }^{2} 4 e_{2}{ }^{2}\right)\end{array}\end{array}$} & \multicolumn{6}{|c|}{$\begin{array}{c}\text { Isomer II } \\
\left(1 a_{1}{ }^{2} 1 e^{4} 1 b_{2}{ }^{2} 1 b_{1}{ }^{2} 2 a_{1}{ }^{2} 2 e^{4} 3 e^{4} 3 a_{1}{ }^{2} 2 b_{2}{ }^{2} 1 a_{2}{ }^{2} 4 a_{1}{ }^{2}\right. \\
\left.2 b_{1}{ }^{2} 5 a_{1}{ }^{2} 4 e^{4} 3 b_{2}{ }^{2} 3 b_{1}{ }^{2} 5 e^{4} 6 e^{4} 7 e^{4} 6 a_{1}{ }^{2} 7 a_{1}{ }^{2} 4 b_{2}{ }^{2}\right)\end{array}$} \\
\hline & & \multicolumn{2}{|r|}{ UPBEO $^{\dagger}$} & \multicolumn{2}{|c|}{ UB3LYP $\ddagger$} & \multicolumn{2}{|c|}{ ROCCSD $(T)^{\S}$} & \multicolumn{2}{|r|}{ UPBEO ${ }^{\dagger}$} & \multicolumn{2}{|c|}{ UB3LYP ${ }^{\ddagger}$} & \multicolumn{2}{|c|}{ ROCCSD (T) $)^{\S}$} \\
\hline & & MO & VDE (theo.) & MO & VDE (theo.) & MO & VDE (theo.) & MO & VDE (theo.) & MO & VDE (theo.) & MO & VDE (theo.) \\
\hline$x$ & $2.71(5)$ & $4 e_{2}$ & 2.58 & $4 e_{2}$ & 2.49 & $4 e_{2}$ & 2.59 & $4 b_{2}$ & 2.53 & $4 b_{2}$ & 2.47 & $4 b_{2}$ & 2.61 \\
\hline$A$ & 3.45 (3) & $2 b_{2}$ & 2.97 & $2 b_{2}$ & 2.91 & $2 b_{2}$ & 3.28 & $7 a_{1}$ & 3.09 & $7 a_{1}$ & 3.02 & $7 a_{1}$ & -11 \\
\hline$B$ & 3.78 (3) & & - & & - & & -11 & & - & & - & & -11 \\
\hline C & $4.86(5)$ & & - & & - & & -11 & & - & & - & & -11 \\
\hline$D$ & $5.3(1)$ & & - & & - & & -11 & & - & & - & & -11 \\
\hline$E$ & $5.6(1)$ & & - & & - & & -11 & & - & & - & & -11 \\
\hline & Es were & es in & te the uncertainti & $\begin{array}{l}(2 \mathrm{df}) \\
+\mathrm{G}(\end{array}$ & $\begin{array}{l}\text { PBEO/Def2-TZV } \\
\text { //PBEO/Def2-T }\end{array}$ & $\begin{array}{l}\text { vel of } t \\
\text { level }\end{array}$ & $\begin{array}{l}\text { ry. Spin contam } \\
\text { heory, because } t\end{array}$ & on & $\mathrm{V}$ & . & contamination. & & \\
\hline
\end{tabular}

a
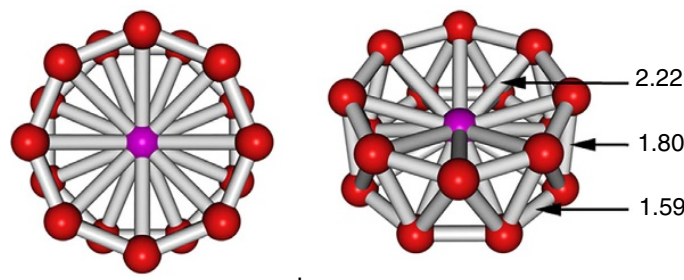

I

$$
\left(D_{8 d},{ }^{3} A_{2}\right)
$$

b
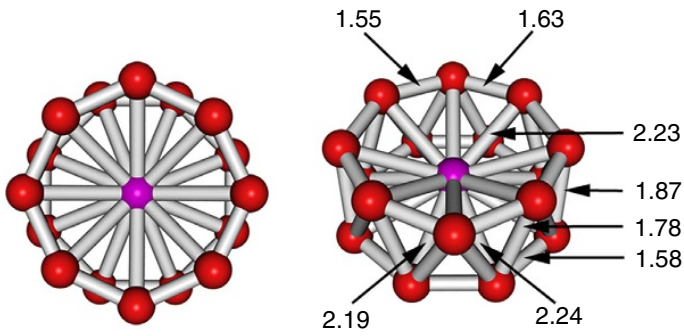

II

$\left(C_{4 v}, A_{1}\right)$

Figure 2 | Two views of isomer I and isomer II of the $\mathrm{CoB}_{\mathbf{1 6}}$ cluster. The point group symmetries and spectroscopic states of isomer I (a) and isomer II (b) are shown in parentheses. Sticks drawn between atoms help visualization and do not necessarily represent classical $2 c-2 e$ B-B or Co-B bonds here and elsewhere. All distances are in $\AA$.

distorted drum-like structure composed of two $\mathrm{B}_{7}$ rings with two $B$ atoms outside the drum (Supplementary Fig. 1). In fact, the majority of the low-lying isomers within $20 \mathrm{kcal} \mathrm{mol}^{-1}$ (Supplementary Fig. 1) represent various derivatives (drum-like or possessing principal geometrical features of the drum-like structure) of isomers I and II, showing the stability of the drumlike structures. It should be noted that there are significant bonding interactions between the two $\mathrm{B}_{8}$ rings and between the Co atom and all $16 \mathrm{~B}$ atoms in both isomers I and II (vide infra). Interestingly, the drum structure in a quintet state (isomer XIV in Supplementary Fig. 1) appears to be the most stable one out of all other quintet isomers. It should be mentioned that there were two previous DFT calculations on similar drum-like structures of neutral boron clusters doped with transition metal atoms ${ }^{24,25}$.

To facilitate comparisons between the experimental and theoretical results, we calculated low-lying VDEs of isomers I and II of $\mathrm{CoB}_{16}^{-}$using three methods (Table 1). We found that the VDEs computed using the two DFT methods are not very impressive; but we observed good agreement between the theoretical VDEs at ROCCSD(T)/6-311 + G(2df) and the experimental data for the first two detachment channels (Table 1). Since isomer $\mathbf{I}$ is open shell, the electron detachment energy from the doubly degenerate $4 \mathrm{e}_{2}$ - $\mathrm{HOMO}$ should lead to a doublet final state for the neutral. The computed VDE at ROCCSD(T) is $2.59 \mathrm{eV}$, compared with the experimental VDE of $2.71 \mathrm{eV}$. The next electron detachment from the non-degenerate $2 b_{2}-\mathrm{HOMO}-1$ should lead to both a quartet and a doublet final state, with the quartet being lower in energy. The calculated VDE for the quartet final state at ROCCSD(T) is $3.28 \mathrm{eV}$, compared with the VDE of the $\mathrm{A}$ band at $3.45 \mathrm{eV}$. Unfortunately, we were not able to calculate any higher VDEs because of the limitation of the ROCCSD(T) method. However, we believe that the good agreement between experiment and theory for the first two VDEs provides sufficient credence for the identified drum-like isomer $\mathbf{I}$ for the $\mathrm{CoB}_{16}^{-}$cluster.

Isomer II gives very similar theoretical VDEs as isomer I at all three levels of theory, consistent with the similarities in their geometries. Since isomer II is a closed shell species, we were able to calculate only the first VDE value at the ROCCSD $(T)$ method as $2.61 \mathrm{eV}$, also in good agreement with the experimental data. Furthermore, the calculated ADEs of isomer $\mathbf{I}(2.45 \mathrm{eV})$ and isomer II $(2.43 \mathrm{eV})(\mathrm{PBE} 0 / \mathrm{Def} 2-\mathrm{TZVP})$ are in excellent agreement with the experimentally measured $\mathrm{ADE}$ value of $2.48 \mathrm{eV}$. We should point out that there is a Jahn-Teller distortion for the neutral $\mathrm{CoB}_{16}$ drum-like structure of isomer $\mathbf{I}$, consistent with the broad X band observed in the PES spectra (Fig. 1). Indeed, the calculated relaxed neutral $\mathrm{CoB}_{16}$ structure $\mathbf{I}^{\mathbf{0}}$ (Supplementary Fig. 2 and Supplementary Table 1$)$ has lower symmetry $\left(C_{2 v}\right)$, as one would expect for the Jahn-Teller distorted structure due to the occupation of the doubly degenerate HOMO $\left(4 \mathrm{e}_{2}\right)$ of isomer I by a single electron. In fact, the HOMO $\left(4 \mathrm{~b}_{2}\right)$ of isomer II originates from the HOMO $\left(4 \mathrm{e}_{2}\right)$ of isomer I when one of the doubly degenerate orbitals is doubly occupied. Therefore, the detachment of one electron from the doubly occupied HOMO $\left(4 b_{2}\right)$ of isomer II leads to the same neutral structure $\mathbf{I}^{\mathbf{0}}$. The high relative energy of isomer III, as well as its appreciably higher theoretical first VDE of $3.65 \mathrm{eV}$ (Supplementary Table 2), makes this cluster unlikely to be populated in the molecular beam in any appreciable amount.

\section{Discussion}

Tubular (or drum-like) boron clusters have been of interest for many years, because they can be considered as the embryos for boron nanotubes ${ }^{14}$. However, such drum-like structures have never 
been observed experimentally for bare boron clusters, even though they have been shown to be stable computationally ${ }^{14,26-29}$. For instance, the $B_{20}$ cluster was first suggested as the global minimum on the basis of theoretical calculations ${ }^{14}$, but it has not been observed or confirmed experimentally ${ }^{29}$. Tubular structures were also studied for the bare $\mathrm{B}_{16}^{+}, \mathrm{B}_{16}, \mathrm{~B}_{16}^{-}$and $\mathrm{B}_{16}^{2-}$ species ${ }^{15,30}$. For the $\mathrm{B}_{16}^{+}$cationic cluster, the tubular structure was suggested to be the global minimum ${ }^{15}$, whereas the tubular structures of both $\mathrm{B}_{16}$ and $\mathrm{B}_{16}^{-}$were found to be high-energy isomers ${ }^{30}$. Clearly, the strong coordination interactions with the Co atom significantly stabilize the tubular $B_{16}$ to give the drum-like global minima (structures I and II) for $\mathrm{CoB}_{16}^{-}$. Bare anionic boron clusters are found to be $2 \mathrm{D}$ up to $\mathrm{B}_{36}^{-}$(ref. 13), while some transitionmetal-doped anionic boron clusters are found to preserve the planar boron framework on metal doping 31,32 . The largest experimentally observed metal-doped boron cluster $\left(\mathrm{CoB}_{12}^{-}\right)$ maintains a similar planar geometry for the $\mathrm{B}_{12}$ moiety ${ }^{32}$. Hence, the doping of the Co atom induces an earlier 2D-to-3D transition for boron clusters, as shown by the $3 \mathrm{D}$ isomers I and II of $\mathrm{CoB}_{16}^{-}$. In fact, the $\mathrm{CoB}_{16}^{-}$drum structure represents the highest coordination number known in chemistry today. The previous highest coordination number known experimentally was 15 for $\left[\mathrm{Th}\left(\mathrm{H}_{3} \mathrm{BNMe}_{2} \mathrm{BH}_{3}\right)_{4}\right]$ (ref. 33), though theoretical studies have suggested the highest coordination numbers of 15 in $\mathrm{PbHe}_{15}^{2+}$ (ref. 34) and 16 in the Friauf-Laves phases in $\mathrm{MgZn}_{2}$ or $\mathrm{MgNi}_{2}$ (ref. 35). Endohedral fullerenes $\left(\mathrm{M} @ \mathrm{C}_{60}\right)$ have been observed ${ }^{36,37}$, but the metal atom in those cases interacts with the $\mathrm{C}_{60}$ shell primarily ionically and it does not stay in the centre of $\mathrm{C}_{60}$.

It is interesting to point out that the $\mathrm{B}-\mathrm{B}$ distances in the $\mathrm{B}_{8}$ rings of both isomers I and II of $\mathrm{CoB}_{16}^{-}$and the bare tubular $\mathrm{B}_{16}$ are very similar (Supplementary Table 3 ). To gain insight into the chemical bonding of the $\mathrm{CoB}_{16}^{-}$drums, we performed chemical bonding analyses for isomers I and II using the Adaptive Natural Density Partitioning (AdNDP) method ${ }^{38}$, which is an extension of the popular Natural Bond Orbital method ${ }^{39}$. It should be noted that the bonding in some double-ring tubular boron clusters has been discussed previously $9,40,41$.

Since isomer I has two unpaired electrons, we used the unrestricted AdNDP (UAdNDP) analysis, which enables treatments of the $\alpha$ and $\beta$ electrons separately. To obtain an averaged result for a bond (Fig. 3), we added the UAdNDP results for the $\alpha$ and $\beta$ electrons of the same type of bonds. According to the UAdNDP analysis results, the 58 valence electrons in $\mathrm{CoB}_{16}^{-}$can be divided into four sets. The first set (Fig. 3a,b) consists of localized bonding elements, while the other three sets (Fig. $3 c-g, h-j, k-o$ ) are composed of delocalized bonding elements. In the first set, the UAdNDP analysis for isomer I revealed the following localized bonding elements: one lone pair (1c-2e bond) (Fig. 3b) of $3 \mathrm{~d}_{\mathrm{z}} 2$ type on Co with an occupation number $(\mathrm{ON})$ of $1.98|\mathrm{e}|$ and sixteen $2 \mathrm{c}-2 \mathrm{e} \mathrm{B}-\mathrm{B} \sigma$-bonds (Fig. 3a) with $\mathrm{ON}=1.84|\mathrm{e}|$ within each $B_{8}$ ring (all superimposed onto the $B_{16}$ fragment in Fig. 3), which can also be viewed as $3 \mathrm{c}-2 \mathrm{e}$ bonds with the $\mathrm{ON}=1.96|\mathrm{e}|$ responsible for the bonding between the boron rings. In the last case, a boron atom from the neighbouring ring contributes somewhat $(0.12|\mathrm{e}|)$ to the formation of the $3 \mathrm{c}-2 \mathrm{e}$ $\sigma$-bond. The $2 \mathrm{c}-2 \mathrm{e} \mathrm{B}-\mathrm{B} \sigma$-bonds are very similar to the peripheral $\mathrm{B}-\mathrm{B}$ bonds found in all $2 \mathrm{D}$ boron clusters ${ }^{8-10}$. The second set includes five delocalized $\sigma$ bonds (denoted as $\sigma+\sigma$ ), which are formed from delocalized $\sigma$ bonds between the two $\mathrm{B}_{8}$ rings. Since the $\sigma$ orbitals between the two boron rings overlap positively, we designate them as $\sigma+\sigma$ in the second set, which constitutes $\sigma$-aromaticity according to the $4 n+2(n=2)$ Hückel rule. The three delocalized $16 \mathrm{c}-2 \mathrm{e} \sigma+\sigma$ bonds (Fig. 3c-e) with $\mathrm{ON}=1.82-1.86|\mathrm{e}|$ involve only $\sigma$-bonding within the boron rings, whereas the two delocalized $17 \mathrm{c}-2 \mathrm{e} \sigma+\sigma$ bonds (Fig. 3f,g) come primarily from the $3 \mathrm{~d}_{\mathrm{xy}}$ and $3 \mathrm{~d}_{\mathrm{x}} 2_{-\mathrm{y}} 2$ AOs of Co interacting with the boron rings. It should be noted that the direct covalent interactions between Co and the $\mathrm{B}_{16}$ unit via the $3 \mathrm{~d}_{\mathrm{xy}}$ and $3 \mathrm{~d}_{\mathrm{x}} 2 \mathrm{y}_{\mathrm{y}} 2$ AOs of Co are found to be around $0.6|\mathrm{e}|$ according to the AdNDP analysis. The third set (Fig. $3 \mathrm{~h}-\mathrm{j}$ ) shows three delocalized $\sigma-\sigma$ bonds, which represent bonding interactions within each ring, but anti-bonding interactions between the two boron rings. This set of delocalized bonds also constitutes $\sigma$-aromaticity according to the $4 n+2(n=1)$ Hückel rule. In the third set, the $16 c-2 \mathrm{e} \sigma-\sigma$ bond (Fig. $3 \mathrm{~h}$ ) involves mainly the two boron rings, whereas the two $17 \mathrm{c}-2 \mathrm{e} \sigma-\sigma$ bonds (Fig. 3i,j) involve interactions between the $3 \mathrm{~d}_{\mathrm{xz}}$ and $3 \mathrm{~d}_{\mathrm{yz}}$ AOs of Co with the boron rings. The direct covalent interaction of the $3 \mathrm{~d}_{\mathrm{xz}}$ and $3 \mathrm{~d}_{\mathrm{yz}}$ AOs of Co with the boron kernel is assessed to be around $0.5|\mathrm{e}|$. The last set includes five delocalized bonds, which represent $\pi-\pi$ interactions between the boron rings: three $16 \mathrm{c}-2 \mathrm{e} \pi-\pi$ bonds (Fig. $3 \mathrm{k}-\mathrm{m}$ ) with $\mathrm{ON}=1.98-2.00|\mathrm{e}|$ and two $16 \mathrm{c}-1 \mathrm{e} \pi-\pi$ bonds (Fig. 3n,o) with $\mathrm{ON}=1.00|\mathrm{e}|$ (one unpaired electron on each bond). The eight $\pi$ electrons in the last set suggest $\pi$-aromaticity according to the $4 n$ rule $(n=2)$ for triplet states. Therefore, the stability of isomer $\mathbf{I}$ of $\mathrm{CoB}_{16}^{-}$can be considered to be due to the double $\sigma$ - and $\pi$-aromaticity and bonding interactions of the $3 \mathrm{~d}$ AOs of Co with the $\mathrm{B}_{8}$ rings.

As expected, isomer II of $\mathrm{CoB}_{16}^{-}$, which is close in energy and geometry to isomer I, has almost the same bonding pattern as that of isomer I (Supplementary Fig. 3). All the bonding elements found in isomer I are also found in isomer II except for the last set (Supplementary Fig. 3k-n). Since isomer II is closed shell, eight electrons in the last set are observed to form four $16 \mathrm{c}-\mathrm{e} \pi-\pi$ bonds with $\mathrm{ON}=1.98-2.00 \quad|\mathrm{e}|$, rendering this isomer $\pi$-antiaromatic. Hence, isomer II exhibits conflicting aromaticity ( $\sigma$-aromatic and $\pi$-antiaromatic), which leads to some distortion to $C_{4 v}$ symmetry compared to the $D_{8 d}$ symmetry of the doubly aromatic isomer $\mathbf{I}$. As was mentioned earlier, the HOMO $\left(4 \mathrm{~b}_{2}\right)$ of isomer II originates from the HOMO $\left(4 \mathrm{e}_{2}\right)$ of isomer I when one of the doubly degenerate orbitals is doubly occupied. Indeed, occupation of only one degenerate MO by two electrons causes the electronic instability, which causes the geometric rearrangement of isomer II lowering the $\mathrm{D}_{8 \mathrm{~d}}$ symmetry to $\mathrm{C}_{4 \mathrm{v}}$.

To understand the interactions between $\mathrm{Co}$ and the tubular $\mathrm{B}_{16}$ host, we have performed AdNDP analyses for the neutral $\mathrm{B}_{16}$ tubular isomer (Supplementary Fig. 4). Similar to isomers I and II of $\mathrm{CoB}_{16}^{-}$, the AdNDP analyses give $162 \mathrm{c}-2 \mathrm{e} \mathrm{B}-\mathrm{B} \sigma$-bonds with $\mathrm{ON}$ values of $1.70|\mathrm{e}|$ within the two $\mathrm{B}_{8}$ rings. The encapsulation of $\mathrm{Co}$ strengthens the $\mathrm{B}-\mathrm{B} \sigma$-bonds within each $\mathrm{B}_{8}$ ring in $\mathrm{CoB}_{16}^{-}$, but weakens the inter-ring interactions, compared with the bare $\mathrm{B}_{16}$, as reflected by their ON values (Fig. 3 and Supplementary Fig. 4) and the B-B bond lengths (Supplementary Table 3). The remaining 16 electrons in $\mathrm{B}_{16}$ participate in delocalized bonding: five $16 c-2 e \sigma+\sigma$ bonds and three $16 c-2 e \pi-\pi$ bonds, rendering the tubular $\mathrm{B}_{16}$ doubly $\sigma$ - and $\pi$-aromatic. The major difference in chemical bonding between the drum-like $\mathrm{B}_{16}$ and $\mathrm{CoB}_{16}^{-}$comes from two factors: (1) the formation of an additional set (Fig. $3 \mathrm{~h}-\mathrm{j}$ ) of the delocalized $\sigma-\sigma$ bonds in $\mathrm{CoB}_{16}^{-}$; and (2) participation of Co $3 \mathrm{~d}$ AOs in the two 17c-2e $\sigma+\sigma$ bonds (Fig. 3f,g). Both factors are consistent with structural changes between $\mathrm{CoB}_{16} \overline{\text { and }}$ $\mathrm{B}_{16}$. There are strong bonding interactions between $\mathrm{Co}$ and the $\mathrm{B}_{16}$ host in $\mathrm{CoB}_{16}^{-}$to stabilize the tubular $\mathrm{B}_{16}$ structure, because the global minimum of $B_{16}$ is planar ${ }^{30}$.

Isomer $\mathbf{I}$ of $\mathrm{CoB}_{16}^{-}$is open shell with two unpaired electrons, whereas isomer II can be viewed as a result of Jahn-Teller distortion from isomer I. Addition of two electrons to isomers I or II would create a closed shell and doubly aromatic $\mathrm{CoB}_{16}^{3-}$ species with $\mathrm{D}_{8 \mathrm{~d}}$ symmetry. Our calculations indeed confirmed this hypothesis: $\mathrm{CoB}_{16}^{3-}$ was found to be a minimum on the potential energy surface with very similar bond distances as in isomer I (Supplementary Table 3). The triply charged $\mathrm{CoB}_{16}^{3-}$ species can be electronically stabilized by external akali metal 
a

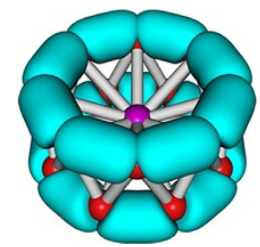

Sixteen 2c-2e B-B $\sigma$ bonds

$\mathrm{ON}=1.84 \mathrm{lel}$ b

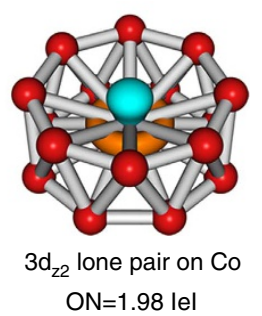

C

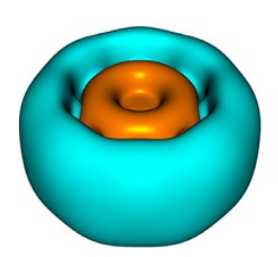

Three $16 \mathrm{c}-2 \mathrm{e} \sigma+\sigma$ bonds

$\mathrm{ON}=1.82-1.86 \mathrm{lel}$

d

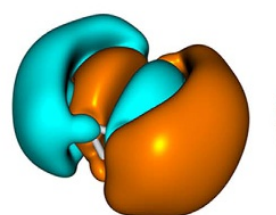

h

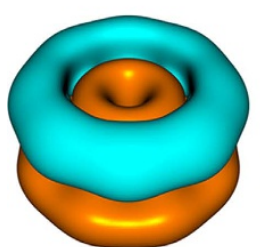

One $16 c-2 e \sigma-\sigma$ bond

$\mathrm{ON}=1.86 \mathrm{lel}$

k

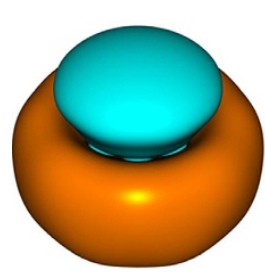

I

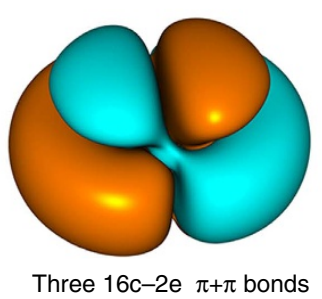

$\mathrm{ON}=1.98-2.00$ lel e

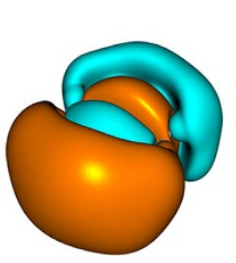

i

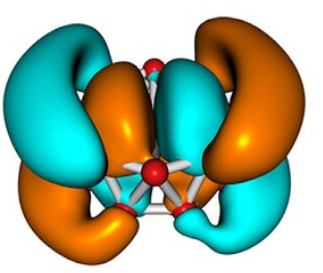

Two 17c-2e $\sigma-\sigma$ bonds

$\mathrm{ON}=2.00 \mathrm{lel}$

j f

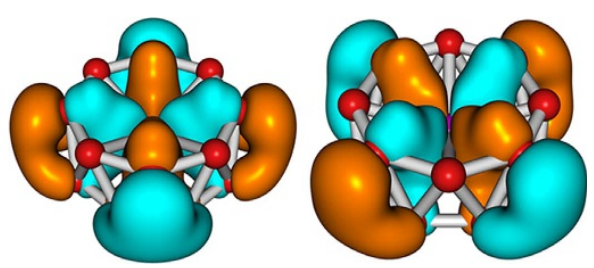

Two 17c-2e $\sigma+\sigma$ bonds

$\mathrm{ON}=2.00 \mathrm{lel}$

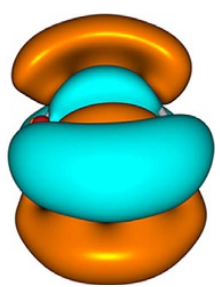

n

m

\section{n}

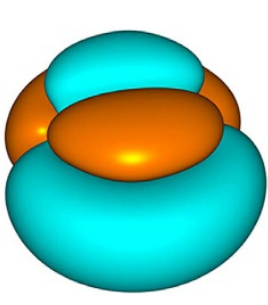

o

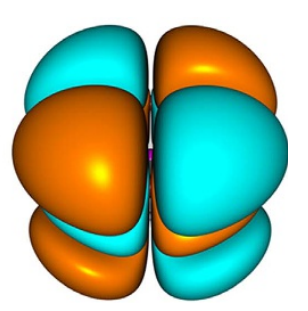

Two $16 c-1$ e $\pi-\pi$ bonds

$\mathrm{ON}=1.00 \mathrm{lel}$

Figure 3 | Chemical bonding picture. (a-o) The overall chemical bonding picture (a-o) obtained for the isomer I of the $\mathrm{CoB}_{16}$ molecular drum via the UAdNDP analysis. ON denotes occupation number here and elsewhere.

cations, such as in $\mathrm{Na}_{2} \mathrm{CoB}_{16}^{-}$. Since ligation would be needed to ultimately synthesize $\mathrm{CoB}_{16}^{-}$, we considered a triple-decked $\left[\mathrm{CoB}_{16}(\mathrm{CaCp})_{2}\right]^{-}$sandwich complex (Supplementary Fig. 5), using the divalent $\mathrm{Ca}$ atoms and the aromatic $\mathrm{C}_{5} \mathrm{H}_{5}^{-}\left(\mathrm{Cp}^{-}\right)$ligands. It should be mentioned that similar $\left[\mathrm{CpLiB}_{6} \mathrm{LiCp}\right]^{2-}$ triple-decked complex ${ }^{42}$ with the double antiaromatic $\mathrm{B}_{6}^{2-}$ unit was previously suggested to be stable and viable experimentally. We found that the $\left[\mathrm{CoB}_{16}(\mathrm{CaCp})_{2}\right]^{-}$ triple-decked complex was a minimum on the potential energy surface with high electronic stability. All the B-B and Co-B bond lengths were found to be almost the same as in isomers I and II of $\mathrm{CoB}_{16}^{-}$(Supplementary Table 3). We have further performed AdNDP analyses and found that the triple-decked sandwich complex exhibits exactly the same chemical bonding pattern as the parent $\mathrm{CoB}_{16}^{-}$(Supplementary Figs 6-8). The Natural Population Analysis (NPA) charge on Ca was found to be +1.54 , consistent with the initial hypothesis and the charge-transfer nature of the triple-decked $\left[\mathrm{CoB}_{16}(\mathrm{CaCp})_{2}\right]^{-}$sandwich complex. Thus, the $\mathrm{CoB}_{16}^{-}$molecular drum can serve as a building block for the design of novel cluster-assembled nanomaterials. The high stability of the $\mathrm{CoB}_{16}^{-}$drum structures may also help the search for new metal-boride phases containing various boron ring units $^{43}$.

We have produced and characterized a large Co-doped boron cluster, $\mathrm{CoB}_{16}^{-}$, using photoelectron spectroscopy and quantumchemical calculations. Extensive computational searches established two high symmetry $\left(\mathrm{D}_{8 \mathrm{~d}}\right.$ and $\left.\mathrm{C}_{4 \mathrm{v}}\right)$ drum-like structures with $\mathrm{Co}$ sandwiched by two $\mathrm{B}_{8}$ rings as nearly degenerate global minima. The $\mathrm{CoB}_{16}^{-}$molecular drums represent the highest coordination for a metal atom known in chemistry and opens new possibilities for designing novel boron-based nanomaterials. First, the $\mathrm{CoB}_{16}^{-}$drums may be considered as the embryo to make filled boron nanotubes due to the significant $\mathrm{B}-\mathrm{B}$ bonding between the two $B_{8}$ rings. Second, there are possibilities to 
observe larger doped-boron clusters with even higher coordination number to further push the limit of coordination number in chemistry. Third, we have demonstrated one possibility to use $\mathrm{CoB}_{16}^{-}$as a building block of new cluster-assembled nanomaterials in a triple-decked complex.

\section{Methods}

Experimental methods. The experiment was carried out using a magnetic-bottle PES apparatus equipped with a laser vaporization cluster source ${ }^{44}$. Briefly, the $\mathrm{CoB}_{16}^{-}$anion clusters were produced by laser vaporization of a cold-pressed target composed of $\mathrm{Co}$ and isotopically enriched ${ }^{11} \mathrm{~B}$. Bismuth was added as a binder and it also provided a convenient calibrant $\left(\mathrm{Bi}^{-}\right)$for the PES experiment. Clusters formed in the nozzle were entrained in a He carrier gas and underwent a supersonic expansion to form a collimated cluster beam. The He carrier gas was seeded with $5 \% \mathrm{Ar}$ for better cooling of the entrained clusters ${ }^{22}$. The anionic clusters were extracted from the collimated cluster beam and analysed by a time-offlight mass spectrometer. The $\mathrm{CoB}_{16}^{-}$anion clusters were mass selected and decelerated before being photodetached by a laser beam at $193 \mathrm{~nm}(6.424 \mathrm{eV})$ from an $\mathrm{ArF}$ excimer laser or $266 \mathrm{~nm}$ (4.661 eV) from a Nd:YAG laser. Photoelectrons were collected at nearly $100 \%$ efficiency by a magnetic bottle and analysed in a $3.5 \mathrm{~m}$ long flight tube. The resolution of the apparatus, $\Delta \mathrm{Ek} / \mathrm{Ek}$, was about than $2.5 \%$, that is, $\sim 25 \mathrm{meV}$ for $1 \mathrm{eV}$ electrons.

Theoretical methods. Search for the global minimum of $\mathrm{CoB}_{16}^{-}$was performed using the Coalescence Kick program ${ }^{45}$ at the PBE0/3-21G level of theory ${ }^{46,47}$. The Coalescence Kick algorithm generated $\sim 10,000$ trial structures for each spin multiplicity (singlet, triplet and quintet), followed by geometry optimization. Low-lying isomers within $25 \mathrm{kcal} \mathrm{mol}^{-1}$ were further refined at a more expansive basis set, Def2-TZVP ${ }^{48}$. For each structure, vibrational frequencies were calculated and imaginary frequencies were followed to ensure that the isomer corresponded to a true minimum on the potential energy surface. Spin contamination was found to be $<10 \%$ in all DFT calculations. For selected isomers, we performed additional geometry optimization at various DFT levels, as well as more accurate single-point coupled-cluster calculations $[\operatorname{ROCCSD}(\mathrm{T}) / 6-311+\mathrm{G}(2 \mathrm{df})]$, to reliably establish the relative energy ordering. Vertical detachment energies of the three lowest energy structures were calculated at three different methods (UPBE0, UB3LYP and $\operatorname{ROCCSD}(\mathrm{T})$ ) to compare with the experimental data. The VDEs were obtained as the difference in energy between the ground state of the anion and selected low-lying electronic states of the neutral molecule at the geometry of the anion. All calculations were done using GAUSSIAN-09 (ref. 49).

To understand the chemical bonding, we carried out electron localization analyses using the AdNDP method ${ }^{38}$ at the PBE0/6-31G(d) level of theory. Previously, AdNDP results have been shown to be insensitive to the level of theory or basis set used ${ }^{50}$. The AdNDP analysis is based on the concept of electron pairs as the main elements of chemical bonds. It represents the molecular electronic structure in terms of $n$-centre two-electron ( $n c-2 \mathrm{e})$ bonds, recovering the familiar lone pairs $(1 \mathrm{c}-2 \mathrm{e})$ and localized $2 \mathrm{c}-2 \mathrm{e}$ bonds or delocalized $n \mathrm{c}-2 \mathrm{e}$ bonds $\left(3 \leq n \leq\right.$ total number of atoms in the system). The MOLEKEL 5.4.0.8 program $^{51}$ is used for molecular structure and AdNDP bond visualizations.

\section{References}

1. Albert, B. \& Hillebrecht, H. Boron: elementary challenge for experimenters and theoreticians. Angew. Chem. Int. Ed. Engl. 48, 8640-8668 (2009).

2. Oganov, A. R. et al. Ionic high-pressure form of elemental boron. Nature 457, 863-867 (2009).

3. Kuhlmann, U. \& Werheit, H. Improved Raman effect studies on boron carbide $\left(\mathrm{B}_{4.3}\right.$ C). Phys. Status Solidi (b) 175, 85-92 (1993).

4. Nelmes, R. J. et al. Observation of inverted-molecular compression in boron carbide. Phys. Rev. Lett. 74, 2268-2271 (1995).

5. Kawai, R. \& Weare, J. H. Instability of the $B_{12}$ icosahedral cluster: rearrangement to a lower energy structure. J. Chem. Phys. 95, 1151-1159 (1991).

6. Boustani, I. A comparative study of $a b$ initio SCF-CI and DFT. Example of small boron clusters. Chem. Phys. Lett. 233, 273 (1995).

7. Hanley, L., Whitten, J. L. \& Anderson, S. L. Collision-induced dissociation and $\mathrm{ab}$ initio studies of boron cluster ions: determination of structures and stabilities. J. Phys. Chem. 92, 5803-5812 (1988).

8. Alexandrova, A. N., Boldyrev, A. I., Zhai, H. J. \& Wang, L. S. All-boron aromatic clusters as potential new inorganic ligands and building blocks in chemistry. Coord. Chem. Rev. 250, 2811-2866 (2006).

9. Sergeeva, A. P. et al. Understanding boron through size-selected clusters: structure, chemical bonding, and fluxionality. Acc. Chem. Res. 47, 1349-1358 (2014).

10. Li, W. L., Pal, R., Piazza, Z. A., Zeng, X. C. \& Wang, L. S. B $B_{27}^{-}$: appearance of the smallest boron cluster containing a hexagonal vacancy. J. Chem. Phys. 142, 204305 (2015).

11. Li, W. L., Zhao, Y. F., Hu, H. S., Li, J. \& Wang, L. S. B- ${ }_{30}^{-}$: A quasiplanar chiral boron cluster. Angew. Chem. Int. Ed. Engl. 53, 5540-5545 (2014).
12. Li, W. L. et al. The $B_{35}$ cluster with a double-hexagonal vacancy: a new and more flexible structural motif for borophene. J. Am. Chem. Soc. 136, 12257-12260 (2014).

13. Piazza, Z. A. et al. Planar hexagonal $B_{36}$ as a potential basis for extended singleatom layer boron sheets. Nat. Commun. 5, 3113 (2014).

14. Kiran, B. et al. Planar-to-tubular structural transition in boron clusters: $B_{20}$ as the embryo of single-walled boron nanotubes. Proc. Natl Acad. Sci. USA 102, 961-964 (2005).

15. Oger, E. et al. Boron cluster cations: transition from planar to cylindrical structures. Angew. Chem. Int. Ed. Engl. 46, 8503-8506 (2007).

16. Chen, Q. et al. Experimental and theoretical evidence of an axially chiral borospherene. ACS Nano. 9, 754-760 (2015).

17. Zhai, H. J. et al. Observation of an all-boron fullerene. Nat. Chem 6, 727-731 (2014).

18. Romanescu, C., Galeev, T. R., Li, W. L., Boldyrev, A. I. \& Wang, L. S. Transition-metal-centered monocyclic boron wheel clusters $\left(M \subset B_{n}\right)$ : a new class of aromatic borometallic compounds. Acc. Chem. Res. 46, 350-358 (2013).

19. Zhai, H. J., Alexandrova, A. N., Birch, K. A., Boldyrev, A. I. \& Wang, L. S. Hepta- and octa-coordinated boron in molecular wheels of 8- and 9-atom boron clusters: observation and confirmation. Angew. Chem. Int. Ed. Engl. 42, 6004-6008 (2003).

20. Galeev, T. R., Romanescu, C., Li, W. L., Wang, L. S. \& Boldyrev, A. I. Observation of the highest coordination number in planar species: decacoordinated $\mathrm{Ta} \subseteq \mathrm{B}_{10}^{-}$and $\mathrm{Nb} \odot \mathrm{B}_{10}^{-}$anions. Angew. Chem. Int. Ed. Engl. 51, 2101-2105 (2012).

21. Zhai, H. J., Wang, L. S., Alexandrova, A. N. \& Boldyrev, A. I. Electronic structure and chemical bonding of $\mathrm{B}_{5}^{-}$and $\mathrm{B}_{5}$ by photoelectron spectroscopy and ab initio calculations. J. Chem. Phys. 117, 7917-7924 (2002).

22. Alexandrova, A. N., Boldyrev, A. I., Zhai, H. J. \& Wang, L. S. Electronic structure, isomerism, and chemical bonding in $\mathrm{B}_{7}^{-}$and $\mathrm{B}_{7}$. J. Phys. Chem. A 108, 3509-3517 (2004).

23. Piazza, Z. A. et al. A photoelectron spectroscopy and ab initio study of $\mathrm{B}_{21}^{-}$: negatively charged boron clusters continue to be planar at 21. J. Chem. Phys. 136, 104310 (2012)

24. Xu, C., Cheng, L. J. \& Yang, J. L. Double aromaticity in transition metal centered double-ring boron clusters $\mathrm{M} @ \mathrm{~B}_{2 \mathrm{n}}(\mathrm{M}=\mathrm{Ti}, \mathrm{Cr}, \mathrm{Fe}, \mathrm{Ni}, \mathrm{Zn} ; \mathrm{n}=6,7,8)$. J. Chem. Phys. 141, 124301 (2014).

25. Tam, N. M., Pham, H. T., Duong, L. V., Pham-Ho, M. P. \& Nguyen, M. T. Fullerene-like boron clusters stabilized by an endohedrally doped iron atom: $\mathrm{B}(\mathrm{n}) \mathrm{Fe}$ with $\mathrm{n}=14,16,18$ and 20. Phys. Chem. Chem. Phys. 17, 3000-3003 (2015).

26. An, W., Bulusu, S., Gao, Y. \& Zeng, X. C. Relative stability of planar versus double-ring tubular isomers of neutral and anionic boron cluster $\mathrm{B}_{20}$ and $\mathrm{B}_{20}^{-}$. J. Chem. Phys. 124, 154310 (2006).

27. Sergeeva, A. P. et al. $\mathrm{B}_{22}^{-}$and $\mathrm{B}_{23}^{-}$: all-boron analogues of anthracene and phenanthrene. J. Am. Chem. Soc. 134, 18065-18073 (2012).

28. Popov, I. A., Piazza, Z. A., Li, W. L., Wang, L. S. \& Boldyrev, A. I. A combined photoelectron spectroscopy and $a b$ initio study of the quasi-planar $\mathrm{B}_{24}^{-}$cluster. J. Chem. Phys. 139, 144307 (2013).

29. Romanescu, C., Harding, D. J., Fielicke, A. \& Wang, L. S. Probing the structures of neutral boron clusters using IR/VUV two color ionization: $B_{11}, B_{16}$, and $B_{17}$. J. Chem. Phys. 137, 014317 (2012).

30. Sergeeva, A. P., Zubarev, D. Y., Zhai, H.-J., Boldyrev, A. I. \& Wang, L. S. A photoelectron spectroscopic and theoretical study of $\mathrm{B}_{16}^{-}$and $\mathrm{B}_{16}^{2-}$ : an all-boron naphthalene. J. Am. Chem. Soc. 130, 7244-7246 (2008).

31. Li, W. L., Romanescu, C., Piazza, Z. A. \& Wang, L. S. Geometrical requirements for transition-metal-centered aromatic boron wheels: the case of $\mathrm{VB}_{10}^{-}$. Phys. Chem. Chem. Phys. 14, 13663-13669 (2012).

32. Popov, I. A., Li, W. L., Piazza, Z. A., Boldyrev, A. I. \& Wang, L. S. Complexes between planar boron clusters and transition metals: a photoelectron spectroscopy and ab initio study of $\mathrm{CoB}_{12}^{-}$and $\mathrm{RhB}_{12}^{-}$. J. Phys. Chem. A 118, 8098-8105 (2014).

33. Daly, S. R. et al. Synthesis and properties of a fifteen-coordinate complex: the thorium aminodiboranate $\left[\mathrm{Th}\left(\mathrm{H}_{3} \mathrm{BNMe}_{2} \mathrm{BH}_{3}\right)_{4}\right]$. Angew. Chem. Int. Ed. Engl 49, 3379-3381 (2010).

34. Hermann, A., Lein, M. \& Schwerdtfeger, P. The Gregory-Newton problem of kissing sphere applied to chemistry: the search for the species with the highest coordination number. Angew. Chem. Int. Ed. Engl. 46, 2444-2447 (2007).

35. Komura, Y. \& Tokunaga, K. Structural studies of stacking variants in Mg-base Friauf-Laves phases. Acta Crystallogr. Sect. B 36, 1548-1554 (1980).

36. Wang, L. S. et al. The electronic structure of $\mathrm{Ca}_{6} \mathrm{C}_{60}$. Chem. Phys. Lett. 207, 354-359 (1993).

37. Popov, A. A., Yang, S. \& Dunsch, L. Endohedral fullerenes. Chem. Rev. 113, 5989-6113 (2013).

38. Zubarev, D. Y. \& Boldyrev, A. I. Developing paradigms of chemical bonding: adaptive natural density partitioning. Phys. Chem. Chem. Phys. 10, 5207-5217 (2008). 
39. Foster, J. P. \& Weinhold, F. Natural bond orbitals. J. Am. Chem. Soc. 102, 7211-7218 (1980)

40. Yuan, Y. \& Cheng, L. J. $\mathrm{B}_{14}^{2+}$ : a magic number double-ring cluster. J. Chem. Phys. 137, 044308 (2012).

41. Johansson, M. P. On the strong ring currents in $\mathrm{B}_{20}$ and neighboring boron toroids. J. Phys. Chem. C. 113, 524-530 (2009).

42. Yang, L.-M., Wang, J., Ding, Y.-H. \& Sun, C.-C. Sandwich-like compounds based on bare all-boron cluster $\mathrm{B}_{6}^{2-}$. Phys. Chem. Chem. Phys. 10, 2316-2320 (2008).

43. Fokwa, B. P. T. \& Hermus, M. All-boron planar $\mathrm{B}_{6}$ ring in the solid-state phase $\mathrm{Ti}_{7} \mathrm{Rh}_{4} \mathrm{Ir}_{2} \mathrm{~B}_{8}$. Angew. Chem. Int. Ed. Engl. 51, 1702-1705 (2012).

44. Wang, L. S., Cheng, H. S. \& Fan, J. W. Photoelectron spectroscopy of sizeselected transition metal clusters: $\mathrm{Fe}_{n}^{-}, n=3-24$. J. Chem. Phys 102, 9480-9493 (1995).

45. Sergeeva, A. P., Averkiev, B. B., Zhai, H. J., Boldyrev, A. I. \& Wang, L. S. Allboron analogues of aromatic hydrocarbons: $\mathrm{B}_{17}^{-}$and $\mathrm{B}_{18}^{-}$. J. Chem. Phys. 134, 224304 (2011).

46. Adamo, C. \& Barone, V. Toward reliable density functional methods without adjustable parameters: The PBE0 model. J. Chem. Phys. 110, 6158-6170 (1999).

47. Binkley, J. S., Pople, J. A. \& Hehre, W. J. Self-consistent molecular orbital methods. 21. Small split-valence basis sets for first-row elements. J. Am. Chem. Soc. 102, 939-947 (1980).

48. Weigend, F. \& Ahlrichs, R. Balanced basis sets of split valence, triple zeta valence and quadruple zeta valence quality for $\mathrm{H}$ to $\mathrm{Rn}$ : design and assessment of accuracy. Phys. Chem. Chem. Phys. 7, 3297-3305 (2005).

49. Frisch, M. J. et al. GAUSSIAN09, Revision B.01 (Gaussian, Inc., 2009).

50. Sergeeva, A. P. \& Boldyrev, A. I. The chemical bonding of $\mathrm{Re}_{3} \mathrm{Cl}_{9}$ and $\mathrm{Re}_{3} \mathrm{Cl}_{9}^{2-}$ revealed by the adaptive natural density partitioning analyses. Comm. Inorg. Chem. 31, 2-12 (2010).

51. Varetto, U. MOLEKEL 5.4.0.8 (Swiss National Supercomputing Centre, 2009).

\section{Acknowledgements}

This work was supported by the National Science Foundation (CHE-1263745 to L.S.W and CHE-1361413 to A.I.B.). Computer, storage and other resources from the Division of Research Computing in the Office of Research and Graduate Studies at Utah State University are gratefully acknowledged.

\section{Author contributions}

L.S.W. and A.I.B. designed the research. I.A.P. and A.I.B performed and analyzed the calculations. L.S.W., T.J. and G.V.L. designed experiments and analysed the experimental data. All authors contributed to the interpretation and discussion of the data. I.A.P. and T.J. wrote the manuscript.

\section{Additional information}

Supplementary Information accompanies this paper at http://www.nature.com/ naturecommunications

Competing financial interests: The authors declare no competing financial interests.

Reprints and permission information is available online at http://npg.nature.com/ reprintsandpermissions/

How to cite this article: Popov, I. A. et al. Cobalt-centred boron molecular drums with

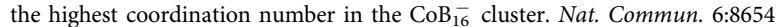
doi: $10.1038 /$ ncomms9654 (2015)

\section{(c) (i)}

This work is licensed under a Creative Commons Attribution 4.0 International License. The images or other third party material in this article are included in the article's Creative Commons license, unless indicated otherwise in the credit line; if the material is not included under the Creative Commons license, users will need to obtain permission from the license holder to reproduce the material. To view a copy of this license, visit http://creativecommons.org/licenses/by/4.0/ 